\title{
Discovery of New Tetracyclic Tetrahydrofuran Derivatives as Potential Broad-Spectrum Psychotropic Agents. ${ }^{\dagger}$
}

Javier Fernández, ${ }^{a,{ }^{*}}$ José M. Alonso, ${ }^{a}$ José I. Andrés, ${ }^{a}$ José M. Cid, ${ }^{a}$ Adolfo Díaz, ${ }^{a}$ Laura Iturrino, ${ }^{a}$ Pilar Gil, $^{a}$ Anton Megens, ${ }^{b}$ Victor K. Sipido, ${ }^{b}$ Andrés A. Trabanco ${ }^{a}$

Johnson \& Johnson Pharmaceutical Research \& Development

a a division of Janssen-Cilag, Medicinal Chemistry Dept., Jarama s/n, 45007 Toledo, Spain.

${ }^{\mathrm{b}}$ a division of Janssen Pharmaceutica N. V., Turnhoutseweg 30, B2340 Beerse, Belgium.

Submitted for the Janssen Memorial Issue

Corresponding author: Tel.: +34-925-24-5770; fax: +34-925-24-5771; e-mail: jfernan0@prdes.jnj.com 


\section{Experimental Section}

General Methods. Reaction solvents were commercially purchased from Aldrich and used without further purification. Commercial reagents were used as received. Reaction were monitored by thinlayer chromatography (TLC) on $0.25 \mathrm{~mm}$ precoated Merck Silica Gel $60 \mathrm{~F}_{254}$, visualizing with ultraviolet light or phosphomolibdic acid stain. Elemental analysis are within $\pm 0.4 \%$ of the theoretical values. ${ }^{1} \mathrm{H}$ NMR spectra were recorded on a Bruker DPX-400 with standard pulse sequences, operating at $400 \mathrm{MHz}$. Chemical shifts $(\delta)$ are reported in parts per million (ppm) downfield from tetramethylsilane (TMS), which was used as internal standard. HPLC-MS analysis were performed with an Agilent Technologies 1100 series consisted of a quaternary pump with degasser, autosampler, column oven and DAD detector. A generic gradient: 80/10/10 of $\mathrm{AcONH}_{4}(0.05 \%) / \mathrm{MeOH} / \mathrm{CH}_{3} \mathrm{CN}$ to $50 / 50 \mathrm{CH}_{3} \mathrm{CN} / \mathrm{MeOH}$ in $6 \mathrm{~min}$, to $100 \% \mathrm{CH}_{3} \mathrm{CN}$ in 1.5 min was performed on a Zorbax XDB C-18, $30 \mathrm{x}$ 4.6mm, i.d. 3.5um from Agilent Technologies. Low-resolution mass spectra (ESI/MS) were recorded on a single quadrupole Micromass Platform series II mass spectrometer with electrospray ionization (ESI). High-resolution mass spectra (HRMS) were recorded on a Micromass LCT Time of Fligh mass spectrometer with electrospray ionization and lockmass device for mass calibration. GC-EI-MS analysis were carried on an Agilent 6890 Series gas chromatograph, with split-splitless injector, directly coupled to an Agilent 5973 mass-selective detector with EI source. GC capillary column was an HP 5-MS (30 mx $0.25 \mathrm{~mm}$ i.d., 0,25 $\mu \mathrm{m}$; Agilent Technologies). Flash column chromatography was performed on Merck Silica Gel 60 (230-400 mesh) using reagent grade heptane, dichloromethane and ACS-grade ethyl acetate and methanol. Preparative HPLC Normal Phase separations were carried on a Waters Delta Prep 4000 with a 5 cm i.d. Prochrom column packed with 200 gr Kromasil $60 \AA$ A 10 um. Enantiomeric separations were performed by HPLC chromatography using a Waters Alliance 2690 
instrument with chiral columns (Chiralcel OD, Chiralcel OJ, Chiralpak AD and Chiralpak AS, Daicel 10um).

\section{Experimental procedures:}

\section{Preparation of $\alpha$-allyl ketones 3a-d.}

2-Fluoro-10-(2-propenyl)-dibenz[b,f]oxepin-11(10H)one (3a): Under nitrogen atmosphere, to a suspension of sodium hydride $(84 \mathrm{mg}, 3.5 \mathrm{mmol})$ in dry THF $(40 \mathrm{~mL})$ at $0{ }^{\circ} \mathrm{C}$ was added dropwise the tricyclic ketone 2a $(685 \mathrm{mg}, 3 \mathrm{mmol})$ in dry THF $(20 \mathrm{~mL})$. The mixture was stirred until hydrogen evolution ceased and the reaction mixture became homogeneous (ca. $2 \mathrm{~h}$ ). Addition of allyl bromide $(0.47 \mathrm{~mL}, 3.05 \mathrm{mmol})$ was followed by stirring of the reaction mixture for $12 \mathrm{~h}$ at room temperature. Aqueous ammonium chloride solution was added $(15 \mathrm{~mL})$, and the resulting mixture was extracted with diethyl ether $(3 \times 50 \mathrm{~mL})$. The combined organic extracts were washed with water and dried over anhydrous sodium sulfate. Volatiles were evaporated in vacuo and the residue thus obtained was purified by flash column chromatography on silica gel (heptane) to give the $\alpha$-allylated ketone 3a: $683 \mathrm{mg}, 85 \%$ yield, white solid, $R_{\mathrm{f}}=0.75$ (heptane); ${ }^{1} \mathrm{H} \mathrm{NMR}\left(\mathrm{CDCl}_{3}\right) \delta 2.78(\mathrm{~m}, 1 \mathrm{H}), 3.05(\mathrm{~m}, 1 \mathrm{H})$, $4.29(\mathrm{t}, 1 \mathrm{H}, J=7.6 \mathrm{~Hz}), 5.04(\mathrm{dd}, 1 \mathrm{H}, J=10.2,1.0 \mathrm{~Hz}), 5.11(\mathrm{dd}, 1 \mathrm{H}, J=17.0,1.2 \mathrm{~Hz}), 5.83(\mathrm{~m}, 1$ H), $7.26(\mathrm{~m}, 5 \mathrm{H}), 7.36(\mathrm{dd}, 1 \mathrm{H}, J=8.9,4.6 \mathrm{~Hz}), 7.70(\mathrm{td}, 1 \mathrm{H}, J=10.2,3.4 \mathrm{~Hz})$; ESI/MS: $269(\mathrm{M}+$ $\mathrm{H})^{+}$

$\alpha$-allyl ketones 3b-d were prepared from allyl bromide and the appropriate tricyclic ketone $\mathbf{2 b}-\mathbf{d}$ according to the procedure described above for compound $\mathbf{3 a}$ :

2-Chloro-10-(2-propenyl)-dibenz[b,f]oxepin-11(10H)one (3b): $657 \mathrm{mg}, 77 \%$ yield, yellow oil, $R_{\mathrm{f}}$ $=0.77$ (heptane); ${ }^{1} \mathrm{H}$ NMR $\left(\mathrm{CDCl}_{3}\right) \delta 2.77(\mathrm{~m}, 1 \mathrm{H}), 3.04(\mathrm{~m}, 1 \mathrm{H}), 4.26(\mathrm{t}, 1 \mathrm{H}, J=7.5 \mathrm{~Hz}), 5.04(\mathrm{dq}$, 
$1 \mathrm{H}, J=9.0,1.5 \mathrm{~Hz}), 5.11(\mathrm{dq}, 1 \mathrm{H}, J=17.0,1.5 \mathrm{~Hz}), 5.82(\mathrm{~m}, 1 \mathrm{H}), 7.15-7.30(\mathrm{~m}, 5 \mathrm{H}) 7.48(\mathrm{dd}, 1 \mathrm{H}$, $J=8.7,2.9 \mathrm{~Hz}), 7.98(\mathrm{~d}, 1 \mathrm{H}, J=2.8 \mathrm{~Hz})$; HRMS calcd for $\mathrm{C}_{17} \mathrm{H}_{14} \mathrm{ClO}_{2} 285.0682$, found 285.0677.

2-Bromo-10-(2-propenyl)-dibenz[b,f]oxepin-11(10H)one (3c): $395 \mathrm{mg}, 40 \%$ yield, colorless oil, $R_{\mathrm{f}}=0.75$ (heptane); ${ }^{1} \mathrm{H}$ NMR $\left(\mathrm{CDCl}_{3}\right) \delta 2.76(\mathrm{~m}, 1 \mathrm{H}), 3.03(\mathrm{~m}, 1 \mathrm{H}), 4.25(\mathrm{t}, 1 \mathrm{H}, J=7.5 \mathrm{~Hz}), 5.04$ $(\mathrm{dd}, 1 \mathrm{H}, J=9.9,0.9 \mathrm{~Hz}), 5.10(\mathrm{dd}, 1 \mathrm{H}, J=17.3,0.9 \mathrm{~Hz}), 5.82(\mathrm{~m}, 1 \mathrm{H}), 7.25(\mathrm{~m}, 5 \mathrm{H}), 7.61(\mathrm{ddd}, 1$ $\mathrm{H}, J=8.7,2.7,0.4 \mathrm{~Hz}), 8.12(\mathrm{~d}, 1 \mathrm{H}, J=2.5 \mathrm{~Hz})$; HRMS calcd for $\mathrm{C}_{17} \mathrm{H}_{14} \mathrm{BrO}_{2} 330.0255$, found 330.0257.

2-Fluoro-10-(2-propenyl)-dibenzo[b,f]tiepin-11(10H)one (3d): $768 \mathrm{mg}, 90 \%$ yield, yellow oil, $R_{\mathrm{f}}$ $=0.68$ (heptane); ${ }^{1} \mathrm{H}$ NMR $\left(\mathrm{CDCl}_{3}\right) \delta 2.85(\mathrm{~m}, 1 \mathrm{H}), 3.27(\mathrm{~m}, 1 \mathrm{H}), 4.93(\mathrm{dd}, 1 \mathrm{H}, J=8.1,6.6 \mathrm{~Hz}), 5.04$ (dd, $1 \mathrm{H}, J=10.2,1.3 \mathrm{~Hz}), 5.14(\mathrm{dd}, 1 \mathrm{H}, J=17.2,1.7 \mathrm{~Hz}), 5.84(\mathrm{~m}, 1 \mathrm{H}), 7.17(\mathrm{~m}, 2 \mathrm{H}), 7.38(\mathrm{~d}, 1 \mathrm{H}$, $J=7.0 \mathrm{~Hz}), 7.44(\mathrm{ddd}, 1 \mathrm{H}, J=8.3,7.8,1.0 \mathrm{~Hz}), 7.58(\mathrm{dd}, 1 \mathrm{H}, J=8.7,5.1 \mathrm{~Hz}), 7.67(\mathrm{~d}, 1 \mathrm{H}, J=7.6$ $\mathrm{Hz}$ ), 7.87 (dd, $1 \mathrm{H}, J=9.9,3.0 \mathrm{~Hz}$ ); HRMS calcd for $\mathrm{C}_{17} \mathrm{H}_{14} \mathrm{FOS} 285.0749$, found 285.0744.

\section{Preparation of cis- $\beta$-allylic alcohols 4a-d.}

\section{cis-10,11-Dihydro-2-fluoro-10-(2-propenyl)-dibenz $[\boldsymbol{b}, \boldsymbol{f}]$ oxepin-11-ol (4a): To a THF $(30 \mathrm{~mL})$}

solution of the $\alpha$-allyl ketone 3a $(537 \mathrm{mg}, 2 \mathrm{mmol})$, cooled at $-30{ }^{\circ} \mathrm{C}, \mathrm{L}$-selectride $(2 \mathrm{ml}$ of $1.0 \mathrm{M}$ solution in THF, $2 \mathrm{mmol}$ ) was added dropwise. The resulting mixture was further stirred and allowed to slowly warm to room temperature for $6 \mathrm{~h}$. Then $2 \mathrm{~N} \mathrm{HCl}(10 \mathrm{~mL})$ was added, and the organic materials were extracted with ethyl acetate $(2 \times 50 \mathrm{~mL})$. The combined organic extracts were washed with brine, dried $\left(\mathrm{Na}_{2} \mathrm{SO}_{4}\right)$, concentrated, and purified by flash column chromatography on silica gel (heptane/ethyl acetate, 4:1) to give cis- $\beta$-allyl alcohol 4a: $497 \mathrm{mg}$, $92 \%$ yield, colorless oil, $R_{\mathrm{f}}=0.75$ (heptane); ${ }^{1} \mathrm{H} \mathrm{NMR}\left(\mathrm{CDCl}_{3}\right) \delta 1.59(\mathrm{~d}, 1 \mathrm{H}, J=9.8 \mathrm{~Hz}), 2.57(\mathrm{~m}, 1 \mathrm{H}), 2.71(\mathrm{~m}, 1 \mathrm{H}), 3.69$ (ddd, $1 \mathrm{H}, J$ 
$=8.5,8.3,1.8 \mathrm{~Hz}), 5.02(\mathrm{dd}, 1 \mathrm{H}, J=9.8,1.8 \mathrm{~Hz}), 5.11(\mathrm{~d}, 1 \mathrm{H}, J=10.2 \mathrm{~Hz}), 5.16(\mathrm{dd}, 1 \mathrm{H}, J=17.3$, 1.4 Hz), $5.88(\mathrm{~m}, 1 \mathrm{H}), 6.94$ (ddd, $1 \mathrm{H}, J=8.9,7.4,3.0 \mathrm{~Hz}), 7.13-7.29(\mathrm{~m}, 6 \mathrm{H})$; HRMS calcd for $\mathrm{C}_{17} \mathrm{H}_{16} \mathrm{FO}_{2} 271.1134$, found 271.1129.

cis- $\beta$-allylic alcohols $\mathbf{4 b}$-d were prepared from the appropriate $\alpha$-allyl ketone ketone $\mathbf{3 b}$-d according to the procedure described above for compound $\mathbf{4 a}$ :

cis-10,11-Dihydro-2-chloro-10-(2-propenyl)-dibenz[b,f]oxepin-11-ol (4b): $516 \mathrm{mg}, 90 \%$ yield, colorless oil, $R_{\mathrm{f}}=0.75$ (heptane); ${ }^{1} \mathrm{H}$ NMR $\left(\mathrm{CDCl}_{3}\right) \delta 1.57(\mathrm{~d}, 1 \mathrm{H}, J=10.8 \mathrm{~Hz}), 2.59(\mathrm{~m}, 1 \mathrm{H}), 2.71$ $(\mathrm{m}, 1 \mathrm{H}), 3.67(\mathrm{t}, 1 \mathrm{H}, J=6.8 \mathrm{~Hz}), 4.99(\mathrm{~d}, 1 \mathrm{H}, J=9.5 \mathrm{~Hz}), 5.11(\mathrm{~d}, 1 \mathrm{H}, J=10.3 \mathrm{~Hz}), 5.16(\mathrm{dd}, 1 \mathrm{H}$, $J=17.2,1.3 \mathrm{~Hz}), 5.87(\mathrm{~m}, 1 \mathrm{H}), 7.2(\mathrm{~m}, 6 \mathrm{H}), 7.44(\mathrm{~d}, 1 \mathrm{H}, J=2.1 \mathrm{~Hz})$; HRMS calcd for $\mathrm{C}_{17} \mathrm{H}_{16} \mathrm{ClO}_{2}$ 287.0839, found 287.0833 .

cis-10,11-Dihydro-2-bromo-10-(2-propenyl)-dibenz[b,f]oxepin-11-ol (4c): $636 \mathrm{mg}, 96 \%$ yield, colorless oil, $R_{\mathrm{f}}=0.75$ (heptane); ${ }^{1} \mathrm{H} \mathrm{NMR}\left(\mathrm{CDCl}_{3}\right) \delta 1.56(\mathrm{~d}, 1 \mathrm{H}, J=9.8 \mathrm{~Hz}), 2.60(\mathrm{~m}, 1 \mathrm{H}), 2.71(\mathrm{~m}$, $1 \mathrm{H}), 3.67(\mathrm{~m}, 1 \mathrm{H}), 4.99$ (dd, $1 \mathrm{H}, J=9.8,1.7 \mathrm{~Hz}), 5.11$ (broad d, $1 \mathrm{H}, J=10.8 \mathrm{~Hz}$ ), 5.17 (broad d, 1 $\mathrm{H}, J=17.0 \mathrm{~Hz}), 5.86(\mathrm{~m}, 1 \mathrm{H}), 7.09(\mathrm{~d}, 1 \mathrm{H}, J=8.5 \mathrm{~Hz}), 7.15-7.30(\mathrm{~m}, 4 \mathrm{H}), 7.35(\mathrm{dd}, 1 \mathrm{H}, J=8.7,2.3$ $\mathrm{Hz}), 7.60(\mathrm{~d}, 1 \mathrm{H}, J=2.4 \mathrm{~Hz})$; HRMS calcd for $\mathrm{C}_{17} \mathrm{H}_{16} \mathrm{BrO}_{2} 331.0334$, found 331.0328.

cis-10,11-Dihydro-2-fluoro-10-(2-propenyl)-dibenzo[b,f]tiepin-11-ol (4d): $556 \mathrm{mg}, 97 \%$ yield, colorless oil, $R_{\mathrm{f}}=0.75$ (heptane); ${ }^{1} \mathrm{H} \mathrm{NMR}\left(\mathrm{CDCl}_{3}\right) \delta 2.00(\mathrm{~m}, 1 \mathrm{H}), 2.45(\mathrm{~m}, 1 \mathrm{H}), 3.65$ (ddd, $1 \mathrm{H}, J=$ 7.5, 6.4, $1.1 \mathrm{~Hz}), 4.85(\mathrm{dd}, 1 \mathrm{H}, J=17.0,1.9 \mathrm{~Hz}), 4.88(\mathrm{~d}, 1 \mathrm{H}, J=9.7 \mathrm{~Hz}), 5.31(\mathrm{~d}, 1 \mathrm{H}, J=4.6 \mathrm{~Hz})$, $5.65(\mathrm{~m}, 1 \mathrm{H}), 5.87(\mathrm{~d}, 1 \mathrm{H}, J=5.0 \mathrm{~Hz}), 7.08(\mathrm{td}, 1 \mathrm{H}, J=8.3,2.9 \mathrm{~Hz}), 7.2(\mathrm{~m}, 3 \mathrm{H}), 7.34(\mathrm{dd}, 1 \mathrm{H}, J=$ 10.3, $2.9 \mathrm{~Hz}), 7.40(\mathrm{dd}, 1 \mathrm{H}, J=7.7,1.0 \mathrm{~Hz}), 7.50(\mathrm{dd}, 1 \mathrm{H}, J=8.5,5.6 \mathrm{~Hz})$; HRMS calcd for $\mathrm{C}_{17} \mathrm{H}_{16} \mathrm{FOS} 287.0906$, found 287.0900.

Preparation of trans- $\beta$-allylic alcohols 5a-d. 
trans-10,11-Dihydro-2-fluoro-10-(2-propenyl)-dibenz[b,f]oxepin-11-ol (5a): To solution of the cis- $\beta$-allyl alcohol 4a (541 mg, $2 \mathrm{mmol})$ and triphenylphosphine $(1.31 \mathrm{~g}, 10 \mathrm{mmol})$ in $30 \mathrm{ml}$ of THF, cooled at $0{ }^{\circ} \mathrm{C}$, a diisopropyl azodicarboxylate (DIAD, $2.02 \mathrm{~g}, 10 \mathrm{mmol}$ ) was added portionwise. The mixture was stirred until a precipitate was formed. A solution of p-nitrobenzoic acid (735 mg, 4.4 $\mathrm{mmol})$ in THF $(50 \mathrm{~mL})$ was dropwise added and the resulting mixture was further stirred at room temperature for $12 \mathrm{~h}$. The solvent was evaporated under vacuum and the residue thus obtained was filtered through silica gel. The residue thus obtained was taken up in dioxane/water $(1: 1,30 \mathrm{~mL}) \mathrm{and}$ $\mathrm{LiOH}$ (53 mg, $2.2 \mathrm{mmol}$ ) was portionwise added. The reaction mixture was stirred at room temperature for $12 \mathrm{~h}$. Aqueous ammonium chloride solution was added $(10 \mathrm{~mL})$, and the resulting mixture was extracted with ethyl acetate $(3 \times 50 \mathrm{~mL})$. The combined organic extracts were washed with brine, dried $\left(\mathrm{Na}_{2} \mathrm{SO}_{4}\right)$, concentrated, and purified by flash column chromatography on silica gel (heptane/ethyl acetate, 4:1) to give the trans- $\beta$-allyl alcohol 5a: $448 \mathrm{mg}, 83 \%$ yield, colorless oil, $R_{\mathrm{f}}=0.75$ (heptane); ${ }^{1} \mathrm{H}$ NMR $\left(\mathrm{CDCl}_{3}\right) \delta 1.99(\mathrm{~d}, 1 \mathrm{H}, J=8.7 \mathrm{~Hz}), 2.41(\mathrm{~m}, 1 \mathrm{H}), 2.58(\mathrm{~m}, 1 \mathrm{H}), 3.29(\mathrm{~m}, 1 \mathrm{H})$, $4.91(\mathrm{dd}, 1 \mathrm{H}, J=8.8,5.5 \mathrm{~Hz}), 4.98(\mathrm{~m}, 2 \mathrm{H}), 5.75(\mathrm{~m}, 1 \mathrm{H}), 6.97$ (ddd, $1 \mathrm{H}, J=8.9,7.7,3.1 \mathrm{~Hz}), 7.05-$ 7.25 (m, $6 \mathrm{H})$; HRMS calcd for $\mathrm{C}_{17} \mathrm{H}_{16} \mathrm{FO}_{2} 271.1134$, found 271.1133.

trans- $\beta$-allylic alcohols $\mathbf{5 b - d}$ were prepared from the appropriate cis- $\beta$-allylic alcohols $4 \mathbf{4 b - d}$ according to the procedure described above for compound $\mathbf{5 a}$ :

trans-10,11-Dihydro-2-chloro-10-(2-propenyl)-dibenz[b,f]oxepin-11-ol (5b): $401 \mathrm{mg}, 70 \%$ yield, colorless oil, $R_{\mathrm{f}}=0.75$ (heptane); ${ }^{1} \mathrm{H} \mathrm{NMR}\left(\mathrm{CDCl}_{3}\right) \delta 1.89(\mathrm{~d}, 1 \mathrm{H}, J=5.8 \mathrm{~Hz}), 2.38(\mathrm{~m}, 1 \mathrm{H}), 2.54(\mathrm{~m}$, $1 \mathrm{H}), 3.28(\mathrm{~m}, 1 \mathrm{H}), 4.95(\mathrm{~m}, 3 \mathrm{H}), 5.73(\mathrm{~m}, 1 \mathrm{H}), 7.05-7.28(\mathrm{~m}, 6 \mathrm{H}), 7.34(\mathrm{~d}, 1 \mathrm{H}, J=2.7 \mathrm{~Hz})$; HRMS calcd for $\mathrm{C}_{17} \mathrm{H}_{16} \mathrm{ClO}_{2} 287.0839$, found 287.0835 . 
trans-10,11-Dihydro-2-bromo-10-(2-propenyl)-dibenz[b,f]oxepin-11-ol (5c): $503 \mathrm{mg}, 76 \%$ yield, colorless oil, $R_{\mathrm{f}}=0.75$ (heptane); ${ }^{1} \mathrm{H}$ NMR $\left(\mathrm{CDCl}_{3}\right) \delta 1.92(\mathrm{~d}, 1 \mathrm{H}, J=6.0 \mathrm{~Hz}), 2.39(\mathrm{~m}, 1 \mathrm{H}), 2.56(\mathrm{~m}$, $1 \mathrm{H}), 3.29(\mathrm{~m}, 1 \mathrm{H}), 4.96(\mathrm{~m}, 3 \mathrm{H}), 5.74(\mathrm{~m}, 1 \mathrm{H}), 7.06-7.28(\mathrm{~m}, 6 \mathrm{H}), 7.44$ (d, $1 \mathrm{H}, J=2.7 \mathrm{~Hz}) . \mathrm{HRMS}$ calcd for $\mathrm{C}_{17} \mathrm{H}_{16} \mathrm{BrO}_{2} 331.0334$, found 331.0330.

trans-10,11-Dihydro-2-fluoro-10-(2-propenyl)-dibenzo[b,f]tiepin-11-ol (5d): $412 \mathrm{mg}, 72 \%$ yield, colorless oil, $R_{\mathrm{f}}=0.75$ (heptane); ${ }^{1} \mathrm{H} \mathrm{NMR}\left(\mathrm{CDCl}_{3}\right) \delta 2.46(\mathrm{~d}, 1 \mathrm{H}, J=5.4 \mathrm{~Hz}), 2.69(\mathrm{~m}, 1 \mathrm{H}), 2.79(\mathrm{~m}$, $1 \mathrm{H}), 3.25(\mathrm{~m}, 1 \mathrm{H}), 5.07(\mathrm{~m}, 2 \mathrm{H}), 5.65(\mathrm{dd}, 1 \mathrm{H}, J=9.1,5.4 \mathrm{~Hz}), 5.84(\mathrm{~m}, 1 \mathrm{H}), 6.81(\mathrm{td}, 1 \mathrm{H}, J=8.3$, 2.5 Hz), 7.03-7.20 (m, $3 \mathrm{H}), 7.03-7.20(\mathrm{~m}, 3 \mathrm{H}), 7.31(\mathrm{dd}, 1 \mathrm{H}, J=9.8,2.3 \mathrm{~Hz}), 7.43(\mathrm{dd}, 1 \mathrm{H}, J=8.5$, $5.6 \mathrm{~Hz}), 7.46(\mathrm{~d}, 1 \mathrm{H}, J=8.1 \mathrm{~Hz})$; HRMS calcd for $\mathrm{C}_{17} \mathrm{H}_{16} \mathrm{FOS} 287.0906$, found 287.0901.

\section{Preparation of 2-iodomethyl derivatives 6a-d.}

\section{[(2SR,3a $R S, 12 \mathrm{~b} S R)+(2 S R, 3 \mathrm{a} R S, 12 \mathrm{~b} S R)]-11-F l u o r o-2-i o d o m e t h y l-2,3,3 \mathrm{a}, 12 \mathrm{~b}-$}

tetrahydrodibenzo $[\boldsymbol{b}, \boldsymbol{f}]$ furo $[2,3-\boldsymbol{d}]$ oxepin (6a): To a stirred solution of the trans- $\beta$-allyl alcohol 5a $(540.6 \mathrm{mg}, 2 \mathrm{mmol})$ in dry dichloromethane $(20 \mathrm{~mL})$ at room temperature, $\mathrm{IPy}_{2} \mathrm{BF}_{4}$ (bis(pyridine)iodonium(I) tetrafluoroborate, $919 \mathrm{mg}, 2.2 \mathrm{mmol}$ ) was added at once. The resulting mixture was stirred at room temperature for $10 \mathrm{~min}$. Dichloromethane $(100 \mathrm{~mL})$ was added and the resulting solution was successively washed with an aqueous $\mathrm{Na}_{2} \mathrm{~S}_{2} \mathrm{O}_{4}$ saturated solution, brine and water. The organic phase was dried $\left(\mathrm{Na}_{2} \mathrm{SO}_{4}\right)$ and vacuum evaporated, affording a residue that was purified by HPLC chromatography (heptane) to give the 2-iodomethyl derivative 6a as a 1:1 mixture of diastereoisomers: $729 \mathrm{mg}, 93 \%$ yield, white solid, $R_{\mathrm{f}}=0.75$ (heptane); ${ }^{1} \mathrm{H} \mathrm{NMR}\left(\mathrm{CDCl}_{3}\right) \delta 1.98$ (td, $1 \mathrm{H}, J=12.2,10.1 \mathrm{~Hz}), 2.29(\mathrm{~m}, 1 \mathrm{H}), 2.43(\mathrm{~m}, 1 \mathrm{H}), 2.76(\mathrm{ddd}, 1 \mathrm{H}, J=12.2,7.1,5.2 \mathrm{~Hz}), 3.21(\mathrm{dd}, 1$ $\mathrm{H}, J=9.7,7.8 \mathrm{~Hz}), 3.38(\mathrm{~m}, 2 \mathrm{H}), 3.41(\mathrm{dd}, 1 \mathrm{H}, J=9.8,5.3 \mathrm{~Hz}), 3.93(\mathrm{~m}, 2 \mathrm{H}), 4.35(\mathrm{~m}, 2 \mathrm{H}), 5.60$ 
(d, $1 \mathrm{H}, J=8.5 \mathrm{~Hz}), 5.67(\mathrm{~d}, 1 \mathrm{H}, J=8.3 \mathrm{~Hz}), 6.90(\mathrm{~m}, 2 \mathrm{H}), 7.07-7-24(\mathrm{~m}, 12 \mathrm{H})$; GC-EI-MS: 396 $\left(\mathrm{M}^{+}\right)$.

2-iodomethyl derivatives $\mathbf{6 b}$-d were prepared by iodocyclization with $\mathrm{IPy}_{2} \mathrm{BF}_{4}$ from the appropriate trans- $\beta$-allylic alcohol $\mathbf{5 b} \mathbf{b}-\mathbf{d}$ according to the procedure described above for compound $\mathbf{6 a}$ :

(2SR,3aRS,12bSR)-11-Chloro-2-iodomethyl-2,3,3a,12b-tetrahydrodibenzo[b,f]furo[2,3- $d]$ oxepin (6b): $776 \mathrm{mg}, 94 \%$ yield, white solid, $R_{\mathrm{f}}=0.75$ (heptane); ${ }^{1} \mathrm{H}$ NMR $\left(\mathrm{CDCl}_{3}\right) \delta 2.01(\mathrm{~m}, 1 \mathrm{H}), 2.24(\mathrm{~m}$, $1 \mathrm{H}), 2.41(\mathrm{~m}, 1 \mathrm{H}), 2.73(\mathrm{~m}, 1 \mathrm{H}), 3.22(\mathrm{dd}, 1 \mathrm{H}, J=9.6,7.5 \mathrm{~Hz}), 3.51(\mathrm{~m}, 3 \mathrm{H}), 4.11(\mathrm{~m}, 2 \mathrm{H}), 4.47$ (m, $2 \mathrm{H}), 5.64(\mathrm{~d}, 1 \mathrm{H}, J=8.3 \mathrm{~Hz}), 5.58(\mathrm{~d}, 1 \mathrm{H}, J=7.5 \mathrm{~Hz}), 6.93(\mathrm{~m}, 2 \mathrm{H}), 7.12-7-35$ (m, $12 \mathrm{H})$; GCEI-MS: $412\left(\mathrm{M}^{+}\right)$.

$[(2 S R, 3 \mathrm{a} R S, 12 \mathrm{~b} S R)+(2 S R, 3 \mathrm{a} R S, 12 \mathrm{~b} S R)]-11-\mathrm{Bromo}-2-$-iodomethyl-2,3,3a,12btetrahydrodibenzo $[\boldsymbol{b}, f]$ furo $[2,3-d]$ oxepin $(\mathbf{6 c}): 777 \mathrm{mg}, 85 \%$ yield, white solid, $R_{\mathrm{f}}=0.75$ (heptane); ${ }^{1} \mathrm{H}$ NMR $\left(\mathrm{CDCl}_{3}\right) \delta 1.98(\mathrm{~m}, 1 \mathrm{H}), 2.28(\mathrm{~m}, 1 \mathrm{H}), 2.45(\mathrm{~m}, 1 \mathrm{H}), 2.74(\mathrm{~m}, 1 \mathrm{H}), 3.20(\mathrm{dd}, 1 \mathrm{H}, J=9.7$, $7.7 \mathrm{~Hz}), 3.42(\mathrm{~m}, 2 \mathrm{H}), 3.44$ (dd, $1 \mathrm{H}, J=10.1,5.1 \mathrm{~Hz}), 4.01(\mathrm{~m}, 2 \mathrm{H}), 4.37(\mathrm{~m}, 2 \mathrm{H}), 5.69(\mathrm{~d}, 1 \mathrm{H}, J=$ $8.3 \mathrm{~Hz}), 5.58(\mathrm{~d}, 1 \mathrm{H}, J=7.5 \mathrm{~Hz}), 6.93(\mathrm{~m}, 2 \mathrm{H}), 7.02-7-29(\mathrm{~m}, 12 \mathrm{H})$; GC-EI-MS: $456\left(\mathrm{M}^{+}\right)$.

$([(2 S R, 3 \mathrm{a} R S, 12 \mathrm{~b} S R)+(2 S R, 3 \mathrm{a} R S, 12 \mathrm{~b} S R)]-11-F l u o r o-2-i o d o m e t h y l-2,3,3 \mathrm{a}, 12 \mathrm{~b}-$ tetrahydrodibenzo $[\boldsymbol{b}, \boldsymbol{f}] \mathbf{f u r o}[\mathbf{2 , 3 - \boldsymbol { d } ]}]$ tiepin $(\mathbf{6 d}): 775 \mathrm{mg}, 94 \%$ yield, pale yellow solid, $R_{\mathrm{f}}=0.75$ (heptane); ${ }^{1} \mathrm{H}$ NMR $\left(\mathrm{CDCl}_{3}\right) \delta 2.05(\mathrm{~m}, 1 \mathrm{H}), 2.38(\mathrm{~m}, 1 \mathrm{H}), 2.55(\mathrm{~m}, 1 \mathrm{H}), 2.82(\mathrm{~m}, 1 \mathrm{H}), 3.25-3.55(\mathrm{~m}$, $6 \mathrm{H}), 4.26(\mathrm{~m}, 1 \mathrm{H}), 4.33(\mathrm{~m}, 1 \mathrm{H}), 5.64(\mathrm{~d}, 1 \mathrm{H}, J=10.8 \mathrm{~Hz}), 5.66(\mathrm{~d}, 1 \mathrm{H}, J=10.6 \mathrm{~Hz}), 6.79(\mathrm{~m}, 2$ H), $7.05(\mathrm{~m}, 4 \mathrm{H}), 7.20(\mathrm{~m}, 2 \mathrm{H}), 7.35(\mathrm{~m}, 4 \mathrm{H}), 8.52(\mathrm{~d}, 1 \mathrm{H}, J=4.1 \mathrm{~Hz}), 8.65(\mathrm{~d}, 1 \mathrm{H}, J=5.2 \mathrm{~Hz})$; GC-EI-MS: $412\left(\mathrm{M}^{+}\right)$.

\section{Preparation of 2-N,N-dimethylamino detivatives 7a-d and 8a-d.}




\section{$(2 S R, 3 \mathrm{a} R S, 12 \mathrm{~b} S R)-2-\mathrm{N}, \mathrm{N}-\mathrm{Dimethylaminomethyl-11-fluoro-2,3,3a,12b-}$}

tetrahydrodibenzo $[b, f]$ furo $[2,3-d]$ oxepin $\quad(7 a)$ and $\quad(2 R S, 3 a R S, 12 b S R)-2-N, N-D i m e t h y l a m i n o$ methyl-11-fluoro-2,3,3a,12b-tetrahydrodibenzo $[b, f]$ furo[2,3- $d]$ oxepin $(8 a)$ : To a solution of the corresponding 2-iodomethyl derivative 6a $(594 \mathrm{mg}, 1.5 \mathrm{mmol})$ in $20 \mathrm{~mL}$ of THF, $\mathrm{CaO}(841 \mathrm{mg}, 15$ mmol) and $4 \mathrm{~mL}$ of a $2 \mathrm{M}$ solution of dimethyamine in THF were added at room temperature. The resulting reaction mixture was heated at $120^{\circ} \mathrm{C}$ (oil bath temperature) into a pressure reactor vessel for 12 h. After cooling to rt the solids were filtered off and the organic solution was evaporated. The resudue thus obtained was taken up with dichloromethane $(50 \mathrm{~mL})$ and was successively washed with an aqueous $\mathrm{Na}_{2} \mathrm{CO}_{3}$ saturated solution and water. The organic extract was dried $\left(\mathrm{Na}_{2} \mathrm{SO}_{4}\right)$ and vacuum concentrated affording a residue that was purified by HPLC chromatography on silica gel $\left(\mathrm{CH}_{2} \mathrm{Cl}_{2} / \mathrm{MeOH}\left(\mathrm{NH}_{3}\right), 98: 2\right)$ yielding the corresponding diastereoisomers 7a and 8a. 7a: $140 \mathrm{mg}, 30 \%$ yield, colorless oil, $R_{\mathrm{f}}=0.32\left(\mathrm{CH}_{2} \mathrm{Cl}_{2} / \mathrm{MeOH}\left(\mathrm{NH}_{3}\right)\right.$ 95:5); ${ }^{1} \mathrm{H} \mathrm{NMR}\left(\mathrm{CDCl}_{3}\right) \delta 1.94(\mathrm{~m}, 1 \mathrm{H}), 2.23(\mathrm{~s}, 6$ H), $2.50(\mathrm{~m}, 2 \mathrm{H}), 2.70(\mathrm{~m}, 1 \mathrm{H}), 3.31(\mathrm{~m}, 1 \mathrm{H}), 4.42(\mathrm{~m}, 1 \mathrm{H}), 4.92(\mathrm{~d}, 1 \mathrm{H}, J=10.8 \mathrm{~Hz})$, 7.05-7.30 (m, $7 \mathrm{H}$ ); HRMS calcd for $\mathrm{C}_{19} \mathrm{H}_{21} \mathrm{FNO}_{2}$ 314.1556, found 314.1558. 8a: $164 \mathrm{mg}$, 35\% yield, colorless oil, $R_{\mathrm{f}}=0.25\left(\mathrm{CH}_{2} \mathrm{Cl}_{2} / \mathrm{MeOH}\left(\mathrm{NH}_{3}\right), 95: 5\right) ;{ }^{1} \mathrm{H} \mathrm{NMR}\left(\mathrm{CDCl}_{3}\right) \delta 2.24(\mathrm{~s}, 6 \mathrm{H}), 2.35(\mathrm{~m}, 1 \mathrm{H}), 2.42(\mathrm{~m}, 1$ H), $2.43(\mathrm{~m}, 2 \mathrm{H}), 3.27(\mathrm{~m}, 1 \mathrm{H}), 4.38(\mathrm{~m}, 1 \mathrm{H}), 4.87(\mathrm{~d}, 1 \mathrm{H}, J=10.8 \mathrm{~Hz}), 7.10(\mathrm{~m}, 3 \mathrm{H}), 7.18(\mathrm{dd}, 1$ $\mathrm{H}, J=8.3,1.3 \mathrm{~Hz}), 7.24(\mathrm{~m}, 3 \mathrm{H})$; HRMS calcd for $\mathrm{C}_{19} \mathrm{H}_{21} \mathrm{FNO}_{2} 314.1556$, found 314.1559.

2-N,N-dimethyl derivatives $\mathbf{7 b - d}$ and $\mathbf{8 b - d}$ were prepared from the appropriate 2-iodomethyl derivative $\mathbf{6 b}-\mathbf{d}$ according to the procedure described above for compounds $7 \mathbf{a}$ and $\mathbf{8 a}$ :

\section{(2SR,3aRS,12bSR)-2-N,N-Dimethylaminomethyl-11-chloro-2,3,3a,12b-}

tetrahydrodibenzo $[\boldsymbol{b}, \boldsymbol{f}]$ furo[2,3- $\boldsymbol{d}]$ oxepin $(\mathbf{7 b}): 186 \mathrm{mg}, 41 \%$ yield, colorless oil, $R_{\mathrm{f}}=0.25$ $\left(\mathrm{CH}_{2} \mathrm{Cl}_{2} / \mathrm{MeOH}\left(\mathrm{NH}_{3}\right), 95: 5\right) ;{ }^{1} \mathrm{H} \mathrm{NMR}\left(\mathrm{CDCl}_{3}\right) \delta 2.36(\mathrm{~m}, 1 \mathrm{H}), 2.45(\mathrm{~m}, 1 \mathrm{H}), 2.53(\mathrm{~s}, 6 \mathrm{H}), 2.79(\mathrm{~m}, 2$ H), $3.40(\mathrm{~m}, 1 \mathrm{H}), 4.49(\mathrm{~m}, 1 \mathrm{H}), 4.90(\mathrm{~d}, 1 \mathrm{H}, J=10.4 \mathrm{~Hz}), 7.06(\mathrm{~d}, 1 \mathrm{H}, J=8.4 \mathrm{~Hz}), 7.08(\mathrm{~m}, 1 \mathrm{H})$, 
$7.20(\mathrm{~m}, 3 \mathrm{H}), 7.33(\mathrm{dd}, 1 \mathrm{H}, J=8.4,2.5 \mathrm{~Hz}), 7.64(\mathrm{~d}, 1 \mathrm{H}, J=2.5 \mathrm{~Hz})$; HRMS calcd for $\mathrm{C}_{19} \mathrm{H}_{21} \mathrm{ClNO}_{2}$ 330.1261 , found 330.1265 .

$(2 S R, 3 \mathrm{a} R S, 12 \mathrm{~b} S R)-2-\mathrm{N}, \mathrm{N}-D i m e t h y l a m i n o m e t h y l-11-b r o m o-2,3,3 \mathrm{a}, 12 \mathrm{~b}-$

tetrahydrodibenzo $[\boldsymbol{b}, f]$ furo $[2,3-d]$ oxepin $(\mathbf{7 c}): 161 \mathrm{mg}, 28 \%$ yield, colorless oil, $R_{\mathrm{f}}=0.19$ $\left(\mathrm{CH}_{2} \mathrm{Cl}_{2} / \mathrm{MeOH}\left(\mathrm{NH}_{3}\right), 95: 5\right) ;{ }^{1} \mathrm{H} \mathrm{NMR}\left(\mathrm{CDCl}_{3}\right) \delta 2.37(\mathrm{~m}, 1 \mathrm{H}), 2.46(\mathrm{~m}, 1 \mathrm{H}), 2.52(\mathrm{~s}, 6 \mathrm{H}), 2.80(\mathrm{~m}, 2$ H), $3.41(\mathrm{~m}, 1 \mathrm{H}), 4.48(\mathrm{~m}, 1 \mathrm{H}), 4.89(\mathrm{~d}, 1 \mathrm{H}, J=10.5 \mathrm{~Hz}), 7.05(\mathrm{~d}, 1 \mathrm{H}, J=8.5 \mathrm{~Hz}), 7.09(\mathrm{~m}, 1 \mathrm{H})$, $7.18(\mathrm{~m}, 3 \mathrm{H}), 7.33(\mathrm{dd}, 1 \mathrm{H}, J=8.5,2.5 \mathrm{~Hz}), 7.63(\mathrm{~d}, 1 \mathrm{H}, J=2.5 \mathrm{~Hz}) ; \mathrm{HRMS}$ calcd for $\mathrm{C}_{19} \mathrm{H}_{21} \mathrm{BrNO}_{2}$ 374.0756, found 374.0760 .

(2SR,3aRS,12bSR)-2-N,N-Dimethylaminomethyl-11-fluoro-2,3,3a,12btetrahydrodibenzo $[\boldsymbol{b}, f]$ furo[2,3- $\boldsymbol{d}]$ tiepin $(\mathbf{7 d}): 196 \mathrm{mg}, 40 \%$ yield, colorless oil, $R_{\mathrm{f}}=0.26$ $\left(\mathrm{CH}_{2} \mathrm{Cl}_{2} / \mathrm{MeOH}\left(\mathrm{NH}_{3}\right), 95: 5\right) ;{ }^{1} \mathrm{H} \mathrm{NMR}\left(\mathrm{CDCl}_{3}\right) \delta 2.07(\mathrm{~m}, 1 \mathrm{H}), 2.36(\mathrm{~s}, 6 \mathrm{H}), 2.53(\mathrm{dd}, 1 \mathrm{H}, J=12.6$, 4.1 Hz), $2.65(\mathrm{dd}, 1 \mathrm{H}, J=12.6,7.2 \mathrm{~Hz}), 2.77(\mathrm{~m}, 1 \mathrm{H}), 3.50(\mathrm{~m}, 1 \mathrm{H}), 4.52(\mathrm{~m}, 1 \mathrm{H}), 5.66(\mathrm{~d}, 1 \mathrm{H}, J=$ $10.1 \mathrm{~Hz}), 6.86(\mathrm{~m}, 1 \mathrm{H}), 7.16(\mathrm{~m}, 3 \mathrm{H}), 7.37(\mathrm{~m}, 2 \mathrm{H}), 7.45(\mathrm{dd}, 1 \mathrm{H}, J=8.4,5.3 \mathrm{~Hz})$; HRMS calcd for $\mathrm{C}_{19} \mathrm{H}_{21} \mathrm{FNOS} 330.1328$, found 330.1331 .

(2RS,3aRS,12bSR)-2-N,N-Dimethylaminomethyl-11-chloro-2,3,3a,12btetrahydrodibenzo $[\boldsymbol{b}, \boldsymbol{f}] \mathbf{f u r o}[\mathbf{2 , 3}-\boldsymbol{d}]$ oxepin $(\mathbf{8 b}): 142 \mathrm{mg}, 29 \%$ yield, colorless oil, $R_{\mathrm{f}}=0.22$ $\left(\mathrm{CH}_{2} \mathrm{Cl}_{2} / \mathrm{MeOH}\left(\mathrm{NH}_{3}\right), 95: 5\right) ;{ }^{1} \mathrm{H} \mathrm{NMR}\left(\mathrm{CDCl}_{3}\right) \delta 2.44(\mathrm{~m}, 2 \mathrm{H}), 2.49(\mathrm{dd}, 1 \mathrm{H}, J=12.9,6.4 \mathrm{~Hz}), 2.59$ (dd, $1 \mathrm{H}, J=12.8,6.3 \mathrm{~Hz}), 3.37(\mathrm{~m}, 1 \mathrm{H}), 4.46(\mathrm{~m}, 1 \mathrm{H}), 4.94(\mathrm{~d}, 1 \mathrm{H}, J=10.8 \mathrm{~Hz}), 7.09$ (m, $2 \mathrm{H})$, $7.18(\mathrm{~m}, 4 \mathrm{H}), 7.52(\mathrm{dd}, 1 \mathrm{H}, J=2.5,0.9 \mathrm{~Hz})$; HRMS calcd for $\mathrm{C}_{19} \mathrm{H}_{21} \mathrm{ClNO}_{2}$ 330.1261, found 330.1263.

$(2 R S, 3 \mathrm{a} R S, 12 \mathrm{~b} S R)-2-\mathrm{N}, \mathrm{N}-D i m e t h y l a m i n o m e t h y l-11-b r o m o-2,3,3 \mathrm{a}, 12 \mathrm{~b}-$ tetrahydrodibenzo $[\boldsymbol{b}, f]$ furo[2,3-d $]$ oxepin $(\mathbf{8 c}): 203 \mathrm{mg}, 36 \%$ yield, colorless oil, $R_{\mathrm{f}}=0.25$ $\left(\mathrm{CH}_{2} \mathrm{Cl}_{2} / \mathrm{MeOH}\left(\mathrm{NH}_{3}\right), 95: 5\right) ;{ }^{1} \mathrm{H} \mathrm{NMR}\left(\mathrm{CDCl}_{3}\right) \delta 2.39(\mathrm{~s}, 6 \mathrm{H}), 2.40(\mathrm{~m}, 2 \mathrm{H}), 2.48(\mathrm{dd}, 1 \mathrm{H}, J=12.8$, 
$6.3 \mathrm{~Hz}), 2.58(\mathrm{dd}, 1 \mathrm{H}, J=12.8,6.4 \mathrm{~Hz}), 3.38(\mathrm{~m}, 1 \mathrm{H}), 4.48(\mathrm{~m}, 1 \mathrm{H}), 4.90(\mathrm{~d}, 1 \mathrm{H}, J=10.7 \mathrm{~Hz}), 7.09$ $(\mathrm{d}, 1 \mathrm{H}, J=8.5 \mathrm{~Hz}), 7.14-7.29(\mathrm{~m}, 4 \mathrm{H}), 7.37(\mathrm{dd}, 1 \mathrm{H}, J=8.6,2.4 \mathrm{~Hz}), 7.60(\mathrm{~d}, 1 \mathrm{H}, J=2.4 \mathrm{~Hz})$; HRMS calcd for $\mathrm{C}_{19} \mathrm{H}_{21} \mathrm{BrNO}_{2} 374.0756$, found 374.0752.

\section{$(2 R S, 3 \mathrm{a} R S, 12 \mathrm{~b} S R)-2-\mathrm{N}, \mathrm{N}-\mathrm{Dimethylaminomethyl-11-fluoro-2,3,3a,12b-}$}

tetrahydrodibenzo $[\boldsymbol{b}, \boldsymbol{f}] \mathbf{f u r o}[\mathbf{2 , 3}-\boldsymbol{d}]$ tiepin $(\mathbf{8 d}): 161 \mathrm{mg}, 32 \%$ yield, colorless oil, $R_{\mathrm{f}}=0.23$ $\left(\mathrm{CH}_{2} \mathrm{Cl}_{2} / \mathrm{MeOH}\left(\mathrm{NH}_{3}\right), 95: 5\right) ;{ }^{1} \mathrm{H} \mathrm{NMR}\left(\mathrm{CDCl}_{3}\right) \delta 2.38(\mathrm{~s}, 6 \mathrm{H}), 2.40-2.57(\mathrm{~m}, 3 \mathrm{H}), 2.63(\mathrm{dd}, 1 \mathrm{H}, J=$ 12.5, $6.5 \mathrm{~Hz}), 3.40(\mathrm{~m}, 1 \mathrm{H}), 4.42(\mathrm{~m}, 1 \mathrm{H}), 5.61(\mathrm{~d}, 1 \mathrm{H}, J=10.5 \mathrm{~Hz}), 6.87(\mathrm{td}, 1 \mathrm{H}, J=8.4,3.0 \mathrm{~Hz})$, $7.10(\mathrm{~m}, 1 \mathrm{H}), 7.17(\mathrm{~m}, 2 \mathrm{H}), 7.38(\mathrm{~m}, 2 \mathrm{H}), 7.45(\mathrm{dd}, 1 \mathrm{H}, J=8.5,5.4 \mathrm{~Hz})$; HRMS calcd for $\mathrm{C}_{19} \mathrm{H}_{21} \mathrm{FNOS} 330.1328$, found 330.1322. Anal. calcd for $\mathrm{C}_{19} \mathrm{H}_{20} \mathrm{FNOS} \mathrm{C,} \mathrm{76.11;} \mathrm{H,} \mathrm{4.88,} \mathrm{O,} \mathrm{11.9,} \mathrm{found}$ C, 76.23; H, 4.97; O, 11.96.

Appendix. Purity determination for intermediates and target compounds: GC, HPLC, ${ }^{1} \mathrm{H}-\mathrm{NMR}$, combustion analysis.

\begin{tabular}{|c|c|c|c|c|c|}
\hline \multirow{2}{*}{ Comp. } & GC- & HPLC- & \multirow{2}{*}{${ }^{1} \mathrm{H}-$} & \multicolumn{2}{|c|}{ Combustion analysis } \\
\cline { 5 - 6 } & purity & purity & NMR & Calculated & Found \\
\hline $\mathbf{3 a}$ & $97 \%$ & $97 \%$ & OK & - & - \\
\hline $\mathbf{3 b}$ & $98 \%$ & $97 \%$ & OK & - & - \\
\hline $\mathbf{3 c}$ & $97 \%$ & $99 \%$ & OK & - & - \\
\hline $\mathbf{3 d}$ & $98 \%$ & $100 \%$ & OK & - & - \\
\hline $\mathbf{4 a}$ & - & $98 \%$ & OK & - & - \\
\hline $\mathbf{4 b}$ & - & $98 \%$ & OK & - & - \\
\hline $\mathbf{4 c}$ & - & $99 \%$ & OK & - & - \\
\hline $\mathbf{4 d}$ & - & $96 \%$ & OK & - & - \\
\hline $\mathbf{5 a}$ & - & $99 \%$ & OK & - & - \\
\hline $\mathbf{5 b}$ & - & $99 \%$ & OK & - & - \\
\hline $\mathbf{5 c}$ & - & $99 \%$ & OK & - & - \\
\hline $\mathbf{5 d}$ & - & $100 \%$ & OK & - & - \\
\hline $\mathbf{6 a}$ & - & $97 \%$ & OK & - & - \\
\hline 6b & - & $100 \%$ & OK & - & - \\
\hline
\end{tabular}




\begin{tabular}{|c|c|c|c|c|c|}
\hline $\mathbf{6 c}$ & - & $99 \%$ & OK & - & - \\
\hline $\mathbf{6 d}$ & - & $98 \%$ & OK & - & - \\
\hline $\mathbf{7 a}$ & - & $98 \%$ & OK & - & - \\
\hline $7 \mathbf{b}$ & - & - & OK & $\begin{array}{c}69.19 \% \mathrm{C}, 6.11 \% \mathrm{H}, \\
4.25 \% \mathrm{~N}\end{array}$ & $\begin{array}{c}69.17 \% \mathrm{C}, 6.12 \% \mathrm{H}, \\
4.23 \% \mathrm{~N}\end{array}$ \\
\hline $\mathbf{7 c}$ & $97 \%$ & $99 \%$ & OK & - & - \\
\hline $\mathbf{7 d}$ & $98 \%$ & $98 \%$ & OK & - & - \\
\hline $\mathbf{8 a}$ & - & - & OK & $\begin{array}{c}72.82 \% \mathrm{C}, 6.43 \% \mathrm{H}, \\
4.47 \% \mathrm{~N}\end{array}$ & $\begin{array}{c}72.80 \% \mathrm{C}, 6.42 \% \mathrm{H}, \\
4.42 \% \mathrm{~N}\end{array}$ \\
\hline $\mathbf{8 b}$ & - & - & OK & $\begin{array}{c}69.19 \% \mathrm{C}, 6.11 \% \mathrm{H}, \\
4.25 \% \mathrm{~N}\end{array}$ & $\begin{array}{c}69.16 \% \mathrm{C}, 6.14 \% \mathrm{H}, \\
4.23 \% \mathrm{~N}\end{array}$ \\
\hline $\mathbf{8 c}$ & $97 \%$ & $99 \%$ & OK & - & - \\
\hline $\mathbf{8 d}$ & - & - & OK & $\begin{array}{c}69.27 \% \mathrm{C}, 6.12 \% \mathrm{H}, \\
4.25 \% \mathrm{~N}\end{array}$ & $\begin{array}{c}69.29 \% \mathrm{C}, 6.11 \% \mathrm{H}, \\
4.26 \% \mathrm{~N}\end{array}$ \\
\hline$(-)-8 d$ & - & - & OK & $\begin{array}{c}69.27 \% \mathrm{C}, 6.12 \% \mathrm{H}, \\
4.25 \% \mathrm{~N}\end{array}$ & $\begin{array}{c}69.20 \% \mathrm{C}, 6.14 \% \mathrm{H}, \\
4.24 \% \mathrm{~N}\end{array}$ \\
\hline$(+)-8 d$ & - & - & OK & $\begin{array}{c}69.27 \% \mathrm{C}, 6.12 \% \mathrm{H}, \\
4.25 \% \mathrm{~N}\end{array}$ & $\begin{array}{c}69.23 \% \mathrm{C}, 6.14 \% \mathrm{H}, \\
4.27 \% \mathrm{~N}\end{array}$ \\
\hline
\end{tabular}

